\title{
Microsurgical treatment for arteriovenous malformation of the corpus callosum and choroidal fissure
}

\author{
Marcos Devanir Silva da Costa, MD, MSc, Bruno Fernandes de O. Santos, MD, MSc, \\ Felipe Bouchabki de Almeida Guardini, MD, and Feres Chaddad-Neto, MD, PhD \\ Department of Neurosurgery, Federal University of São Paulo, Brazil
}

The management of arteriovenous malformations (AVMs) of the corpus callosum and choroidal fissure is challenging because they commonly receive arterial feeders from the anterior and posterior circulation, and drain to deep veins. In this video the authors present the case of a 20 -year-old man who presented with acute onset of headache, loss of consciousness, and nuchal rigidity. Computed tomography, MRI, and cerebral angiography performed in tandem revealed a ruptured, large, Grade IV AVM of the corpus callosum and choroidal fissure with two groups of arterial feeders: one from the pericallosal artery and the other from the medial and lateral posterior choroidal arteries. The treatment strategy included two stages. The first stage involved preoperative embolization of the arterial feeders from the posterior circulation, which promoted reduction of the nidus flow of the AVM. The second involved a microsurgical resection, using the interhemispheric approach, with the patient in the prone position, which allowed accessing the anterior circulation feeders and the complete resection of the AVM, without associated morbidity.

The video can be found here: https://youtu.be/5wcYKhcJtls.

KEYWORDS AVM; supratentorial arteriovenous malformations; corpus callosum; choroidal fissure; microsurgery; preoperative embolization 